\title{
Determining the Supply of Material Resources for High-Rise Construction: Scenario Approach
}

\author{
Anna Minnullina ${ }^{1, *}$ and Vladimir Vasiliev $^{2}$ \\ ${ }^{1}$ State Budget Educational Institution of Higher Education «Industrial University of Tyumen», \\ Volodarskogo str., 38, Tyumen, 625000, Russia \\ ${ }^{2}$ State Budget Educational Institution of Higher Education «Industrial University of Tyumen», \\ Volodarskogo str., 38, Tyumen, 625000, Russia
}

\begin{abstract}
This article presents a multi-criteria approach to determining the supply of material resources for high-rise construction under certain and uncertain conditions, which enables integrating a number of existing models into a fairly compact generalised economic and mathematical model developed for two extreme scenarios.
\end{abstract}

\section{Introduction}

Every year the number of high-rise buildings and their floors is significantly increasing. Constructions around the world become complex, technical and engineering systems, which results in increased technical, environmental, psychological, aesthetic, architectural and other requirements. High-rise erection is closely connected with the active use of intellectual and physical human resources, as well as material resources; pollution and human isolation from the environment. By all means, the above mentioned and many other problems of high-rise construction require a careful study and implementation of the positive results achieved in the course of this research.

Thus, high-rise construction is among the most cost-intensive sectors of economy, with the largest expenses in the sphere of logistics. Due to serious investment in high-rise construction, it is important to correctly determine the supply of material resources. To solve this problem, we have decided to set a multi-criteria problem for determining the economic order quantity of material resources presented for two extreme scenarios.

Nowadays there is a large number of studies and papers related to these problems [1-4], but all these works and approaches examine the objectives in a predominantly logistic format under certain conditions with a simplified justification of the criterion of optimality.

The modern Russian literature on economy often uses the Harris-Wilson model which is related to dynamic deterministic models of inventory management in order to define the economic order quantity (1) $[5-8,16,17]$. This simple classical model helps to understand the movement of stocks and in many cases enables to regulate and control their level.

We calculate the optimal size of supply using the following formula (1):

\footnotetext{
*Corresponding author: minnullinaay@yandex.ru
} 


$$
\bar{N}=\sqrt{\frac{2 \alpha Q}{\beta T}}
$$

where $\alpha$ is shipping and handling expenses as per a delivery lot of material resources;

$T$ is a time period when material resources are used;

$Q$ is a gross requirement of material resources during $T$ time;

$\beta$ is variable expenses for handling a resource unit per unit time.

However, this model is based on certain conditions, i.e. supposed resources are used evenly and as soon as the size of the current stock reaches zero, new supplies are instantly delivered. Demand is regarded as constant in time, and the replenishment of stocks is instant. It is assumed that there is no deficit, therefore only the current stock is considered, the level of which ranges from the maximum value equal to the whole lot at the moment of its receipt to the minimum value equal to zero.

In the classical model of the economic batch quantity, a deficit is not provided but in some cases deficit losses are comparable to the costs of stock maintaining. If there is a deficit, the economic batch quantity model is as follows (2):

$$
\overline{N^{\prime}}=\sqrt{\frac{2 \alpha Q}{\beta}} \times \sqrt{\frac{\beta+\gamma}{\gamma}}
$$

where $\gamma$ is shortage costs of the stock of resource units per unit time.

\section{Methods}

The multi-criteria approach for certain and uncertain conditions we have developed is of a systematic nature and allows integrating a number of existing models into a fairly compact generalised economic and mathematical model presented for two extreme scenarios.

We introduce the following notations for the formal representation of this model:

$l=\overline{1, L}$ is the number of a material resource supplier;

$j=\overline{1, m}$ is a type of the resources used;

$B_{j}$ is a company's demand for the $j$ resource bought;

$A_{l j}$ is the aggregate supply of $j$ resource by $l$ supplier;

$L_{0}^{(j)}=\left(l / A_{l j}>0, l=\overline{1, L}\right)$ is the subset of supplier numbers prioritised for $j$ resource;

$a_{l j}$ is the maximum possible schedule line quantity of $j$ resource by $l$ supplier;

$\alpha_{l j}$ is delivery expenses for the lot of $j$ resources by $l$ supplier;

$\beta_{j}, \gamma_{j}$ are handling and deficit expenses of a $j$ resource unit per unit time (if a deficit is no lower than $\left.\gamma_{j}=+\infty\right)$;

$T_{l j}$ is the schedule date of $j$ resource supplies by $l$ supplier;

$C_{l j}$ is the procurement price of a $j$ resource unit realised by $l$ supplier;

$X_{l j}$ is the supply scope of $j$ resource by $l$ supplier.

\section{Scenario 1}

All the components of supply $(\alpha)$, storage $(\beta)$, deficit $(\gamma)$ and delivery period $(T)$ costs are deterministic. In this case, we represent the multi-criteria definition of the economic order quantity of material resources delivered by suppliers to some construction organisation as the following cluster of $F(x)$ optimality criterion and $S_{1}$ constraint system:

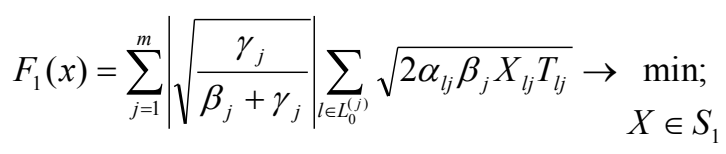




$$
\begin{aligned}
& F_{3}(x)=-\sum_{j=1}^{m} \sum_{l \in L_{0}^{(j)}} \frac{X_{l j}}{\sum_{j \in L_{o}^{(j)}} X_{l j}} \log _{2} \frac{X_{l j}}{\sum_{j \in L_{0}^{(j)}} X_{l j}} \rightarrow \quad \begin{array}{l}
\text { min; } \\
X \in S_{1}
\end{array} \\
& F_{4}(x)=\max _{\substack{1 \leq j \leq m \\
l \in L_{0}^{(i)}}}\left|\sqrt{\frac{2 \alpha_{l j} T_{l j}}{\beta_{j} X_{l j}}\left(\frac{\beta_{j}+\gamma_{j}}{\gamma_{j}}\right)}\right| \rightarrow \begin{array}{l}
\text { min; } \\
X \in S_{1}
\end{array} \\
& F_{5}(x)=\max _{\substack{l, K \in L_{0}^{(j)} \\
l \neq K}}|| \sqrt{\frac{2 \alpha_{l j} X_{l j}}{\beta_{j} T_{l j}}\left(\frac{\beta_{j}+\gamma_{j}}{\gamma_{j}}\right)}|-| \sqrt{\frac{2 \alpha_{k j} X_{k j}}{\beta_{j} T_{k j}}\left(\frac{\beta_{j}+\gamma_{j}}{\gamma_{j}}\right)} \| \rightarrow \min _{X \in S_{1}} j=\overline{1, m} . \\
& S_{1}:\left\{\begin{array}{c}
\sum_{l \in L_{0}^{(j)}} X_{l j} \geq B_{j}, j=\overline{1, m} ; \\
\sum_{l \in L_{0}^{(j)}} X_{l j} \geq \sum_{l \in L_{0}^{(j)}} A_{l j}, j=\overline{1, m} ; \\
\mid \sqrt{\frac{2 \alpha_{l j} X_{l j}}{\beta_{j} T_{l j}}\left(\frac{\beta_{j+\gamma_{J}}}{\gamma_{j}}\right) \mid \leq a_{l j}, l \in L_{0}^{(j)}, j=\overline{1, m} ;} \geq \\
\sum_{l \in L_{0}^{(j)}} \frac{a_{l j} T_{l j}}{\left|\sqrt{\frac{2 \alpha_{l j} T_{l j}}{\beta_{j} X_{l j}}\left(\frac{\beta_{j+\gamma_{J}}}{\gamma_{j}}\right)}\right|} \sum_{l \in L_{0}^{(j)}} A_{l j}, j=\overline{1, m} ; \\
X \geq(\text { or } X \geq 0 \text { and integer - valued }) .
\end{array}\right.
\end{aligned}
$$

\section{Scenario 2}

As a rule, supply $(\alpha)$, storage $(\beta)$, deficit $(\gamma)$ and delivery period $(T)$ expenses are undetermined and can be represented as the following quantities:

$$
\begin{aligned}
& \alpha \in S_{\alpha}:\left\{\alpha_{l j}^{(1)} \leq \alpha_{l j} \leq \alpha_{l j}^{(2)}, j=\overline{1, m}\right\}, \\
& \beta \in S_{\beta}:\left\{\beta_{j}^{(1)} \leq \beta_{j} \leq \beta_{j}^{(2)}, j=\overline{1, m}\right\}, \\
& \gamma \in S_{\gamma}:\left\{\gamma_{j}^{(1)} \leq \gamma_{j} \leq \gamma_{j}^{(2)}, j=\overline{1, m}\right\}, \\
& T \in S_{T}:\left\{T_{l j}^{(1)} \leq T_{l j} \leq T_{l j}^{(2)}, j=\overline{1, m}\right\},
\end{aligned}
$$

where $\left(\alpha^{1}, \alpha^{2}\right),\left(\beta^{1}, \beta^{2}\right),\left(\gamma^{1}, \gamma^{2}\right),\left(T^{1}, T^{2}\right)$ are correspondingly the maximum and minimum values of expense vectors/matrices and resource delivery time. The value changes of $a, A, C$ matrices and $B$ vector are considered to be irrelevant. Otherwise, the system is expanded by $S_{1}, S_{A}, S_{C}, S_{B}$ quantities.

The optimality criterion and constraint system are as follows:

$$
F_{1}(x)=\max _{\alpha \in S_{\alpha}} \sum_{j=1}^{m} \mid \sqrt{\frac{\gamma_{j}}{\beta_{j}+\gamma_{j}}\left|\sum_{i=1}^{n} \sum_{l \in L_{0}^{(j)}}\right| \sqrt{2 \alpha_{l j} \beta_{j} X_{l j} T_{l j}} \mid} \begin{aligned}
& \rightarrow \\
& \beta \in S_{\beta} \\
& \gamma \in S_{\gamma} \\
& T \in S_{T}
\end{aligned}
$$




$$
\begin{aligned}
& F_{3}(x)=-\sum_{j=1}^{m} \sum_{l \in L_{0}^{(j)}} \frac{X_{l j}}{\sum_{j \in L_{o}^{(j)}} X_{l j}} \log _{2} \frac{X_{l j}}{\sum_{j \in L_{0}^{(j)}} X_{l j}} \rightarrow \quad \min ;
\end{aligned}
$$

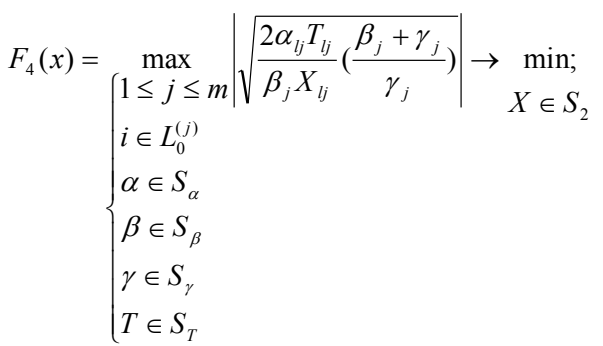

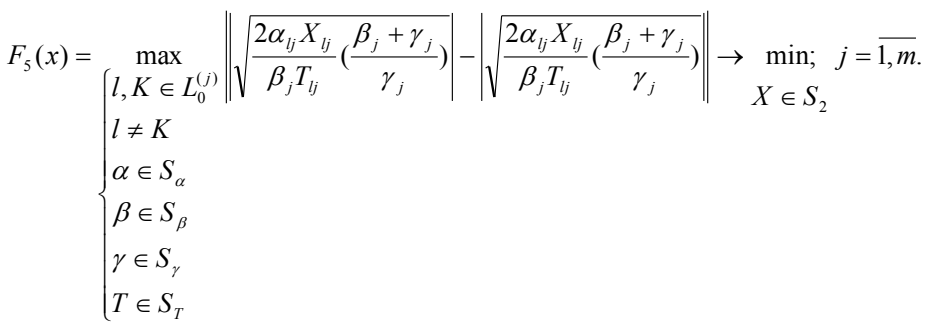

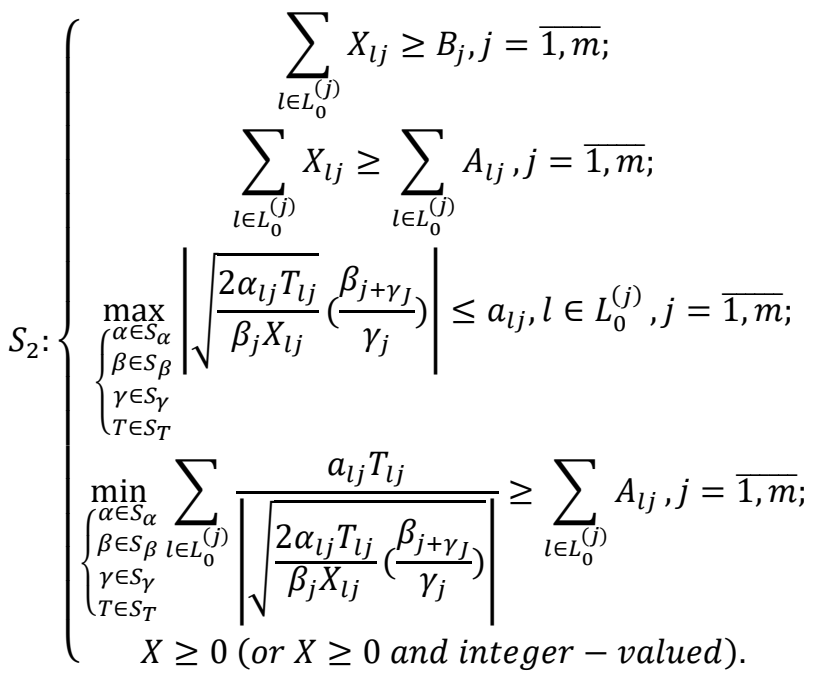

In both scenarios the criteria of optimality represent the following aspects:

$F_{1}(x)$ minimises total production, handling and deficit expenses related to resources;

$F_{2}(x)$ minimises the gross price of the resources bought;

$F_{3}(x)$ minimises the entropy, i.e. the uncertainty of supply quantities;

$F_{4}(x)$ minimises the maximum interval between resource deliveries;

$F_{5}(x)$ minimises the maximum resource delivery tolerance $j=\overline{1, m}$ by various suppliers.

Limitations made by $S_{1}$ constraint region should meet the following criteria:

- demand for each type of resources should be satisfied;

- resource delivery cannot exceed the total offer by all possible suppliers; 
- one delivery lot by suppliers cannot exceed their maximum values;

- the total value of all possible resource deliveries by all possible suppliers is no less than the total offer;

- non-negativity and/or integrality of supplies.

\section{Conclusions}

In case of scenario 2 , the meaning of the optimality criteria and $S_{2}$ constraint system remains the same but the implementation is conducted in the format of the "guaranteed result".

The two model scenarios we presented solve the following problems: realise a multicriteria setting under certain and uncertain conditions; represent an elegant combination of classic models (the transport and Harris-Wilson models); enable to use the well-known mathematical methods for determining the economic order quantity of material resources; allow further development and improvement in various modified forms; have a higher level of adequacy in relation to real-life conditions of construction organisation activities.

Therefore, we can assert that determining the economic order quantity of material resources in high-rise construction should skip the initial data on procurement in the form of criteria for optimal system functioning that limit production conditions and indicators through a kind of filter represented by an economic and mathematical model for making managerial decisions and implementing purchases.

\section{References}

1. A.G. Butrin, Yu.G. Amerhanova, Integratedlogistics, 4, 20 (2009)

2. V.P. Fedko, V.A. Bondarenko, Commerciallogistics, 304 (2006)

3. V.V. Scherbakov, Logistics and Supply Chain Management, 582 (2015)

4. A.A. Kanke, I.P. Koshevaya, Logistics bases, 576 (2013)

5. Yu.G. Lebedev, Logistics harmonized supply chain, 448 (2005)

6. A.P. Tyapukhin, Design of distribution systems based on logistics, 240 (2007)

7. L.B. Mirotin, A.K. Pokrovsky, Fundamentals of Logistics, 192 (2013)

8. M. Linders, F. Johnson, A. Flynn, H. Fearon, Procurement and Supply Management, 752 (2014)

9. D. Bauersoks, D. Kloss,Logistika: integrated supply chain,640(2010)

10. Yu.A. Borodina, O.M. Syardova. Thestrategyofsustainabledevelop18, 56 (2013)

11. A.V. Sysolyatin, Electronic scientific journal13, 1-7(2015)

12. V.S. Lukinskiy, V.V. Lukinskiy, N.G. Pletnev, Logistics and SCM, 360 (2016)

13. V.I. Sergeev, Corporate Logistics, 976 (2008)

14. V.I. Sergeev, I.P. Elyaschevich. SupplyLogistics, 398 (2016)

15. A.Kopytova, Procedia Engineering165, 1132 (2016)

16. M.N. Grigoryev, V.V.Tkach, S.A. Uvarov,Commerciallogistics, 490 (2016)

17. A.Yu. Minnullina,Economics and Entrepreneurship5-2, 734(2014)

18. A.V. Kemenov,Logistics building complex,320 (2012) 